\section{Monika Sawicka, Brasil en el sistema internacional. Roles de la potencia media de nuevo tipo, Universitas, Kraków 2020, pp. 354. ISBN 978-83-242-3652-7, e-ISBN 978-83-242-6488-9}

Anuario Latinoamericano Ciencias Políticas

y Relaciones Internacionales vol. 9, 2020

pp. 221-225

DOI: 10.17951/al.2020.9.221-225

\section{Monika Sawicka, Brazylia w systemie międzynarodowym. Role średniego mocarstwa nowego typu, Universitas, Kraków 2020, pp. 354. ISBN 978-83-242-3652-7, e-ISBN 978-83-242-6488-9}

Monika Sawicka es doctora en ciencias sociales y politóloga, especializada en la investigación de la política interior y exterior de Brasil. Ha publicado varios trabajos científicos en polaco, inglés y portugués, entre otros en la revista $\mathrm{Anu-}$ ario Latinoamericano - Ciencias Políticas y Relaciones Internacionales (Sawicka, 2019). La monografía titulada Brasil en el sistema internacional. Roles de la potencia media de nuevo tipo, publicada en 2020 en polaco, se encuentra en la corriente principal de la investigación politológica en el área de las relaciones internacionales. En la literatura polaca se pueden indicar estudios sobre la actividad internacional de Brasil (Gawrycki, 2013; Gawrycki, 2015), pero el análisis exhaustivo con el enfoque propuesto por la autora amplía significativamente el área de investigación realizada hasta ahora.

Comparto la opinión de que las aspiraciones de las potencias medias de nuevo tipo, combinadas con las nuevas posibilidades creadas por el sistema internacional, que atraviesa un período de transformación, así como los importantes recursos de los que dispone este grupo de países, hacen de las potencias medias un objeto interesante para la investigación en la disciplina de las ciencias políticas (Morales Ruvalcaba, Rocha Valencia, 2015; Sawicka, 2020).

El período de crecimiento de la posición y del papel de Brasil en el sistema internacional, tal como lo analiza la autora, coincide con el gobierno del Partido de los Trabajadores, es decir, dos mandatos presidenciales de Luiz Inácio Lula da Silva (2003-2010) y el primer mandato de la presidenta Dilma Rousseff (2011-2014). Al mismo tiempo el interés de los politólogos por investigar Brasil también ha aumentado.

La autora, con gran erudición, se refiere a adecuadas construcciones teóricas y conocimientos desarrollados en el campo de las ciencias sociales y las 
relaciones internacionales, relevantes en el contexto del tema de investigación.

La estructura del libro se caracteriza por una división proporcional del contenido, corresponde al enfoque adoptado por la autora y le permite presentar una argumentación lógica, de manera coherente y consistente.

En la primera parte del libro, merecen atención y apreciación dos capítulos extensos, en los que la autora revisó y analizó la teoría de los roles internacionales y el concepto del estatus internacional de Estado, señalando correctamente la utilidad analítica de estos enfoques en la investigación que realizó, para luego utilizarlos en el argumento. La autora llevó a cabo un análisis de la categoría teórica del rol del Estado y su uso como herramienta analítica en los estudios de la política exterior estatal basados sobre enfoques constructivistas. Luego, describió la categoría teórica de las potencias medias de nuevo tipo y justificó la adscripción de Brasil a este grupo, basándose en las características constitutivas y conductuales del Estado. Además, analizó los factores que influyen en la formación y redefinición del concepto de los roles nacionales de Brasil y del estatus internacional del Estado.

En la siguiente parte de su argumento, la autora acertadamente se refiere al enfoque constructivista en cuanto a la política exterior de Brasil. La autora analiza la identidad, la importancia de los recursos materiales, las interpretaciones de la propia imagen, las ideas y los valores a los que Brasil se refirió como sujeto activo de las relaciones internacionales en los años 2003-2014.

Al estudiar la dinámica de los roles internacionales de Brasil, tomó en consideración su naturaleza interactiva, las relaciones del Estado con los socios de rol dentro de un contexto específico, condicionado por la estructura del sistema internacional. Para estudiar el proceso de transformación de los roles y percepciones de Brasil se utilizó el concepto de "potencias medias emergentes", es decir, la categoría conceptual de una "potencia media de nuevo tipo", un Estado semiperiférico, una democracia joven, que lucha con el problema de las desigualdades sociales y al mismo tiempo aspira a fortalecer su posición global.

En la segunda parte del libro, en los tres capítulos siguientes, la autora analizó de manera coherente y completa la realización de los tres roles internacionales cuya implementación declaró Brasil. En primer lugar, como un Estado aliado que participa en la construcción de coaliciones globales "en el foro mundial", según lo describe la autora. En segundo lugar, un Estado donante de ayuda al desarrollo que apoya a África en el marco de la diplomacia solidaria. En tercer lugar, un Estado mediador de los conflictos en la región de Oriente Medio.

Utilizando la teoría de los roles internacionales, la autora hizo un análisis constructivista de la política exterior de Brasil. Para la investigación de roles, adoptó un método inductivo, basado en el material seleccionado. Luego, mostrando consistencia en la aplicación del enfoque de investigación declarado, analizó tres roles internacionales, describiendo, analizando y explicando la política exterior de Brasil en 2003-2014. Obviamente, los roles internacio- 
nales analizados no agotan los roles representativos de Brasil en el período analizado. La autora del libro justificó la elección de los tres roles señalados, haciendo referencia a las prioridades de la política exterior del país y la especial importancia que se le asigna a los socios de estos roles, identificada en la etapa del análisis de conformación del concepto de roles.

A fin de refinar el alcance de la investigación, la autora se refirió a varias cuestiones. En primer lugar, los roles internacionales ya señalados e implementados por Brasil para lograr los objetivos marcados por el Estado. En segundo lugar, la identidad, los objetivos y la posición en las relaciones internacionales que atribuyen a Brasil los representantes de la comunidad de política exterior del Estado. En tercer lugar, la forma en que el lugar de Brasil en el sistema internacional en transformación es interpretado y justificado por las élites estatales que componen la comunidad de política exterior, así como por los presidentes y jefes de diplomacia. En cuarto lugar, la conformidad entre de los roles declarados por el Estado con los implementados. En quinto lugar, un conflicto de roles, es decir, un conflicto dentro del rol declarado e implementado por Brasil, con las expectativas por parte de los representantes de la comunidad de política exterior o los socios de rol.

La autora evaluó la coherencia de las expectativas hacia los roles internacionales del Estado, formuladas por la comunidad de política exterior de Brasil, así como la coherencia de los roles declarados e implementados por Brasil y su impacto en el estatus internacional del Estado. Los roles internacionales de Brasil, tal como se identificaron y analizaron en el curso de la argumentación de la autora, permitieron alcanzar los principales objetivos del país, a saber, asegurar un entorno externo propicio para un mayor desarrollo, incluida la expansión económica, aumentar la influencia de Brasil en el proceso de construcción y transformación de las instituciones más importantes de cooperación internacional y llevar al reconocimiento de la posición de Brasil como superpotencia en el sistema internacional. Brasil buscó reconfigurar las instituciones que se dedican a la cooperación económica internacional, ayuda al desarrollo y mediación en los conflictos más importantes que amenazan la seguridad global.

La autora llegó a una conclusión razonable de que la forma en que se declaraban y desempeñaban los roles reflejaba una especie de distanciamiento utilizado por Brasil, resultante de los recursos y la eficiencia del Estado limitados, así como la falta de disposición para aceptar mayores obligaciones internacionales. Según la autora, la elección de roles estuvo determinada por factores culturales, que permitieron a las élites estatales identificar los vínculos de Brasil con los socios de roles, así como factores de carácter prestigioso, el fortalecimiento de la posición del Estado y el aumento de su visibilidad. Como señaló acertadamente la autora, la elección de roles internacionales por parte de Brasil fue determinada por la comprensión de la identidad del Estado y la identificación del lugar que Brasil ocupa y el que desea ocupar en el sistema internacional.
Anuario Latinoamericano Ciencias Políticas y Relaciones Internacionales vol. 9, 2020

pp. 221-225

DOI: 10.17951/al.2020.9.221-225 
En resumen, el análisis de los determinados roles internacionales de Brasil permitió a la autora verificar la validez del supuesto de investigación adoptado de incluir a Brasil en el grupo de las potencias medias de nuevo tipo, que han marcado su presencia en el sistema internacional, esforzándose por redefinir su identidad, posición y rol en las relaciones internacionales contemporáneas. La autora del libro mostró una muy buena comprensión de las teorías, los métodos y las herramientas de investigación en el campo de las ciencias políticas, además de la capacidad de seleccionarlos y utilizarlos adecuadamente. Merece una valoración positiva el hecho de que, sobre la base de los métodos adoptados, haya intentado desarrollar su propio enfoque, teniendo en cuenta la especificidad del tema, el propósito de la investigación y los supuestos de investigación adoptados. Al examinar los roles definidos de Brasil como participante de coaliciones en foros globales, como donante de ayuda al desarrollo para África y como mediador en conflictos en la región de Oriente Medio, analizó los instrumentos políticos, económicos, culturales e ideológicos utilizados por Brasil a nivel regional y suprarregional.

Para examinar la conformación del concepto de los roles y su aplicación, utilizó un análisis cualitativo de los datos existentes y de los datos primarios reunidos mediante entrevistas en profundidad parcialmente estructuradas. También utilizó las técnicas de análisis de datos estadísticos e incluyó cuadros y gráficos útiles que ilustran y enriquecen su argumentación.

También merece atención y aprecio la base de fuentes, extensa, diversa e interdisciplinar, compuesta por publicaciones en varias lenguas, principalmente en portugués e inglés, así como en polaco y español. La lista de fuentes se ha recopilado cuidadosamente por categoría de fuentes utilizadas. Incluye tanto documentos, por ejemplo, de las Naciones Unidas, BRICS o IBSA, como también correspondencia diplomática y otros documentos puestos a disposición de la autora por los Archivos Históricos del Ministerio de Relaciones Exteriores de Brasil, así como informes, guías y estadísticas de, por ejemplo, CEPAL, OPA, OCDE o la OMC. La autora se refirió también a memorias y entrevistas concedidas por presidentes y diplomáticos brasileños, material audiovisual y, por supuesto, monografías y artículos científicos, así como diarios y semanarios brasileños.

El libro se caracteriza por un alto nivel de argumentación sustantiva y un enfoque innovador en el campo de la investigación politológica polaca. Es un primer intento de definir y analizar a Brasil como una potencia media de nuevo tipo, basado en un examen de los roles internacionales de este país durante la presidencia de Luiz Inácio Lula da Silva y Dilma Rousseff, políticos del Partido de los Trabajadores. El valor particular del argumento desarrollado por la autora es el análisis de la política exterior de Brasil, que permitió justificar la clasificación del país como una potencia media de nuevo tipo. Como afirmó acertadamente en su argumento, esto se apoyaba en las características constitutivas del Estado, a saber, la naturaleza no consolidada de una joven democracia, la corrupción endémica, el alto nivel de desigualdades sociales, 
el IDH medio/alto y la posición de la semiperiferia en la economía mundial. La definición de Brasil como una potencia de nuevo tipo también se justifica por los objetivos de la política exterior brasileña encaminados a reconfigurar el orden internacional existente y fortalecer la posición internacional del país. Comparto la opinión de Monika Sawicka de que la actuación de Brasil y los roles internacionales adoptados por el Estado, que la autora definió y analizó, fueron decisivos en este contexto. Un aspecto importante y valioso del análisis de los roles internacionales de Brasil es la comprensión que la autora tiene de sus dinámicas, es decir, cambios en su aplicación como consecuencia de los cambios en la percepción de los socios del rol y las transformaciones de las relaciones mutuas, así como los debates sobre la identidad del Estado y la evolución de la percepción en cuanto a la pertenencia a diferentes grupos de entidades que operan en el sistema internacional.

\section{Referencias bibliográficas}

Gawrycki, M.F. (Ed.) (2013). Brazylia jako mocarstwo wschodzace. Warszawa. Muzeum Historii Polskiego Ruchu Ludowego. Instytut Studiów Iberyjskich i Iberoamerykańskich Uniwersytetu Warszawskiego. ISBN: 978-83-6217-155-2

Gawrycki, M.F. (2015). Latynoamerykańskie koncepcje stosunków międzynarodowych. Tom V. Brazylia. Warszawa. Muzeum Historii Polskiego Ruchu Ludowego. Instytut Studiów Iberyjskich i Iberoamerykańskich Uniwersytetu Warszawskiego. ISBN: 978-83-7901-058-5

Morales Ruvalcaba, D.E., Rocha Valencia, A. (2015). Las promesas del ascenso estructural de los países de América Latina y el Caribe, 1975-2013: logros, desencantos y frustraciones, Anuario Latinoamericano - Ciencias Políticas y Relaciones Internacionales, vol. 2, pp. 185210, DOI: 10.17951/al.2015.2.185

Sawicka, M. (2019). A Tale of Urban Violence in Brazil. The Case of Marielle Franco, Anuario Latinoamericano - Ciencias Políticas y Relaciones Internacionales, vol. 7, pp. 297312, DOI: 10.17951/al.2019.7.297-312

Sawicka, M. (2020). Brazylia w systemie międzynarodowym. Role średniego mocarstwa nowego typu. Universitas, Kraków 2020. ISBN 978-83-242-3652-7, e-ISBN 978-83-2426488-9

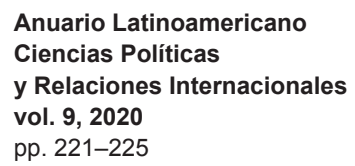

DOI: 10.17951/al.2020.9.221-225

Katarzyna Krzywicka

Facultad de Ciencias Políticas y Periodismo

Universidad Maria Curie-Skłodowska Lublin, Polonia

katarzyna.krzywicka@umcs.edu.pl https://orcid.org/0000-0001-5623-5384 